\title{
Theoretical Studies of Timing and Spectral Properties of Quasars to Nano-quasars till a few $\mathrm{MeV}$
}

\section{Sandip K. Chakrabarti*}

S.N. Bose National Centre for Basic Sciences and Centre for Space Physics

E-mail: chakraba@bose.res.in

\section{Samir Mandal}

Centre for Space Physics

E-mail: samir@csp.res.in

\begin{abstract}
We study the timing and spectral properties of two component accretion flows around black holes which may or may not have jets and outflows. We discuss the origin of quasi-periodic-oscillations (QPOs) as the oscillations of various interfaces, such as the inner edge of the Keplerian disk, shock location, inner shock location as well as due to the periodic cooling of the the subsonic region of the outflow. As far as the spectral properties are concerned, we show that the soft photons emitted by the Keplerian component and the synchrotron radiation from the pre-shock or the post-shock sub-Keplerian component are inverse Comptonized by the thermal and shock-accelerated nonthermal electrons in the post-shock region. The resulting photons can have energy till a few $\mathrm{MeV}$ as observed in several black hole candidates. We produce examples of the spectrum for various flow parameters as well as the mass of the black hole.
\end{abstract}

VI Microquasar Workshop: Microquasars and Beyond

September 18-22 2006

Società del Casino, Como, Italy

\footnotetext{
${ }^{*}$ Speaker.
} 


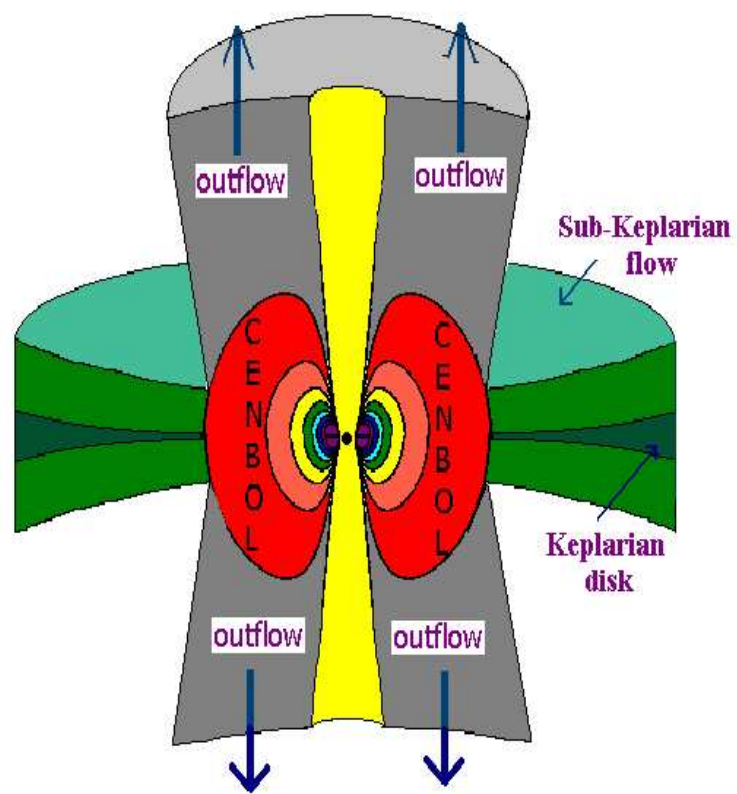

Figure 1: Major constituents of the flow close to a black hole. At a given moment, the emission property depends on which of these components exist. The Keplerian component lies in the equatorial plane and is surrounded by low-angular momentum sub-Keplerian flow. This latter flow may or may not have a centrifugal barrier supported shock wave which behaves like a boundary layer of the black hole (CENBOL). When it is present, it could be steady, oscillating or propagating. The outflow forms out of the CENBOL as long it remains hot. Thus, the spectral properties of the flow, which is dictated by the temperature of the CENBOL is also intimately related to the jet formation.

\section{Introduction}

It has been proven beyond doubt through works of several independent groups that observations of black hole candidates may be best understood when both the Keplerian and sub-Keplerian components are present (e.g., Chakrabarti \& Titarchuk, 1995; Smith et al. 2001;2002). A steady Shakura-Sunyaev (1973) type Keplerian disk cannot explain most of the observational aspects including the spectral state transitions, variability class transitions, quasi-periodic oscillations etc. Though the Shakura-Sunyaev disk was supplemented by a vague component which is sometimes called the corona and sometimes called the base of the jet by various authors, the suggestions themselves are not theoretically justified. They were invoked only because the observations demanded that something like that should exist. On the other hand, the inclusion of the sub-Keplerian component and its accretion shocks by our group as one of the main components of the fbw has been totally justified by numerous theoretical and numerical works. Not surprisingly, the paradigm that emerges out of our work and the cartoon diagrams which emerge out of observational constraints are remarkably converging to the same picture. We present this in Figure 1. Numerical simulations suggest more complex behaviour (Samanta, Ryu and Chakrabarti, 2006) which may be more relevant to explain the observed properties. Our solution appears in the literature under various names, a popular one being the 'truncated Keplerian disk' with a corona. These pseudo-models are generally good enough to 'fit' the data, but they do not provide any intuition regarding physical properties of the system. 
First attempts to incorporate the CENBOL, i.e., shocked sub-Keplerian accretion fbws (see, Chakrabarti 1990 and Abramowicz and Chakrabarti 1990 for the first diagram of the fbw configuration which included a shock) to explain spectral characteristics of quasars was made in Chakrabarti \& Wiita (1992). This was extended to include the Keplerian disk itself, first qualitatively in Chakrabarti (1994) in the context of quasars and subsequently, in the context of stellar mass black holes also by Chakrabarti \& Titarchuk (1995, hereafter CT95) in detail. Here, the soft photons from the Keplerian disk were allowed to be intercepted by the CENBOL and these intercepted photons were reprocessed through inverse Comptonization to produce hard $\mathrm{X}$ rays. Chakrabarti \& Titarchuk (1995) also extended the concept of converging fbws first presented in Blandford and Payne (1982) in the context of black hole accretion and showed that even in the soft state, when the CENBOL is cooled down due to excessive soft photons supply from the Keplerian disk, there may be a faint power-law hard tail due to the Comptonization by bulk-motion of the fbw between the innermost sonic point (which has to be present in any black hole accretion) and the horizon. Unless the magnetic field is very strong, neutron stars should also show this unique effect due to gravitational pull.

However, the synchrotron emission was totally ignored in the past self-consistent works which include sub-Keplerian fbws with shocks, until very recently. Mandal and Chakrabarti (2005, hereafter MC05) included a stochastic magnetic field whose strength is assumed to be proportional to the local equipartition value. Apart from the usual Comptonization by the thermal electrons, MC05 also included the shock acceleration of the electrons and the non-thermal electrons so produced, generated power-law synchrotron photons and at the same time, inverse Comptonize soft photons.

Subsequently, Chakrabarti \& Mandal (2006) included the Keplerian disk also as in CT95, and showed that observations of black hole candidates such as Cyg X-1 can be easily explained by variation of the Keplerian and sub-Keplerian rates. These rates could vary either because of variation of viscosity (which may redistribute matter in sub-Keplerian and Keplerian components) or because of actual changes in the outer boundary. In the present paper, we shall discuss the results from this model. Before we do so, we wish to impress the readers about how easily quasi-periodic oscillations may also be explained using this model.

It is to be noted that if we start with M87 having a mass about a few billion solar mass (Ford et al. 1994; Chakrabarti, 1995) and put it in the category of 'Quasars', then the massive black holes as that in our own galactic center (few million solar mass) would be called 'milli-quasars', the intermediate mass black holes (few hundred to a few thousand times the mass of the Sun) which are behaving like quasars (having collimated relativistic jets) would be micro-quasars and the stellar mass black holes (few to few tens of solar mass) should be called nano-quasars. We will use this terminology in the rest of this article.

\section{Quasi-Periodic Oscillations in Black Holes}

It is generally, and indeed, wrongly believed that the quasi-periodic oscillations (QPOs) have always some thing to do with the inner stable circular orbit (ISCO). The reason why this supposition is false is that, for a given black hole candidate (i.e., for a given ISCO location, and thus frequency of the Keplerian orbit), there can be a wide variation in QPO frequency, often ranging from a few $\mathrm{mHz}$ to a few Hz (see, works of Remillard et al. 1999 and references therein and Chakrabarti et 
al., this volume, specifically for GRO J1655-40). Also, there are several occasions when more than one QPO frequency can be seen simultaneously. The second important fact about QPOs is that it has a significant power (a few percent of $r m s$ value). So it should not be look upon as a 'vibration'. Rather, it is an oscillation of a region (causing it to have a ' $Q$ ' value of around 5 to 40 or so) as a whole. In a two component advective paradigm (Fig. 1), there are several length scales which may participate in 'natural' oscillations. These are: (a) $r=r_{K e p}$ which is the inner edge of the Keplerian disk. This can vary from ISCO when the Keplerian rate is very high and a sub-Keplerian fbw is almost non-existent to a few hundred $r_{g}$, the Schwarzschild radius. So ISCO is useful only when the Keplerian disk extends till ISCO. However, since all the initially Keplerian fbw must pass through the inner sonic point (located between ISCO and marginally bound orbit, see case 'c' below), the fbw deviates from a Keplerian disk well before ISCO. So it is almost never a Keplerian disk with ISCO as the inner edge. (b) $r=r_{s}$ which is the mean location of the shock. This can vary from $\sim 5 r_{g}$ to $100 r_{g}$ or so, depending on specific energy and specific angular momentum. For a Kerr black hole the lower limit could be even closer to the black hole. (c) $r=r_{i n}$, the location of the inner sonic point. This usually varies between the marginally stable and the marginally bound orbits, i.e., between 2 to 3 Schwarzschild radii for a Schwarzschild black hole. For Kerr black holes, both of these orbits are closer to the black hole and thus the frequency can rise. In reality, it is the inner strong shock $r_{\text {in }} \lesssim r_{i s s} \lesssim 10$ with a typical location of $r \sim 7$ just behind $r_{\text {in }}$ is more important (Samanta, Ryu and Chakrabarti, 2006) for the purpose of radiative transfer. This shock forms inside the stronger shock especially when the Rankine-Hugoniot conditions are not satisfied and the fbw is generally non-steady. (d) $r=r_{s r}$, the size of the sonic radius of the outfbw or jet which comes out of the CENBOL. This is usually a few times the shock location (Chakrabarti, 1999; Chakrabarti \& Nandi, 2000). The outfbw till $r_{s r}$ being sub-sonic, it is denser and could be cooled by the soft photons from the disk, especially in the pre-shock fbw $\left(r>r_{s}\right)$. The cold outfbw then falls back and is recycled through the accretion disks again. The four length scales just mentioned produce four time scales and therefore four types of QPOs depending on fbw parameters. The QPO frequency $v$ is is, as a rule of thumb, given by the inverse of the infall time $t_{Q P O}$ from one of the first three types of the oscillating scales $r$ to the horizon, i.e., $t_{Q P O} \sim v^{-1} \sim R r^{3 / 2} 2 G M / c^{3} \mathrm{~s}$, where $R$ is the shock strength $\left(R=1\right.$ for $\left.r=r_{K e p}\right)$ which could be $\sim 7$ for a strong shock and $M$ is the mass of the black hole, be it a quasar or a nano-quasar. For a $M=10 M_{\odot}$ Schwarzschild black hole, for instance, the QPO frequency will be (a) $1.92<v_{a}<1924 \mathrm{~Hz}$ for $3<r<300$, (b) $1.4<v_{b}<127.8 \mathrm{~Hz}$ for $R=7$, and $5<r<100$, (c) $v_{c} \sim 77 \mathrm{~Hz}$ for typical values $\left(R=7, r_{i s s}=7\right.$, $\left.M=10 M_{\odot}\right)$. The fourth type of oscillation depends on the time in which the volume $r_{s r}^{3}$ is filled in till the optical depth $\tau \sim 1$ (Chakrabarti \& Manickam, 2000) is reached. It turns out that this oscillations is of low frequency $(\sim \mathrm{mHz})$ and brings the spectrum from ON to OFF states in quick succession (few minutes) due to local recycling of matter. As Chakrabarti \& Manickam (2000) showed, the duration (recycling time $t_{r e c}$ ) of the QPO, depends on the QPO frequency $v_{b}$ (in the OFF state) itself through the relation $t_{r e c}=v_{d}^{-1} \propto v_{b}^{-2}$. So, $v_{d}$ is not totally independent. The details will be discussed elsewhere.

Since the fbw is turbulent and the absence or presence of shocks strictly depend also on the angular momentum, specific energy, cooling process, it is very difficult to exactly predict all the aspects of QPOs just as it is impossible to predict how a cloud in the autumn sky would evolve in next few minutes or how its albedo is going to alter with time. But needless to mention, the 


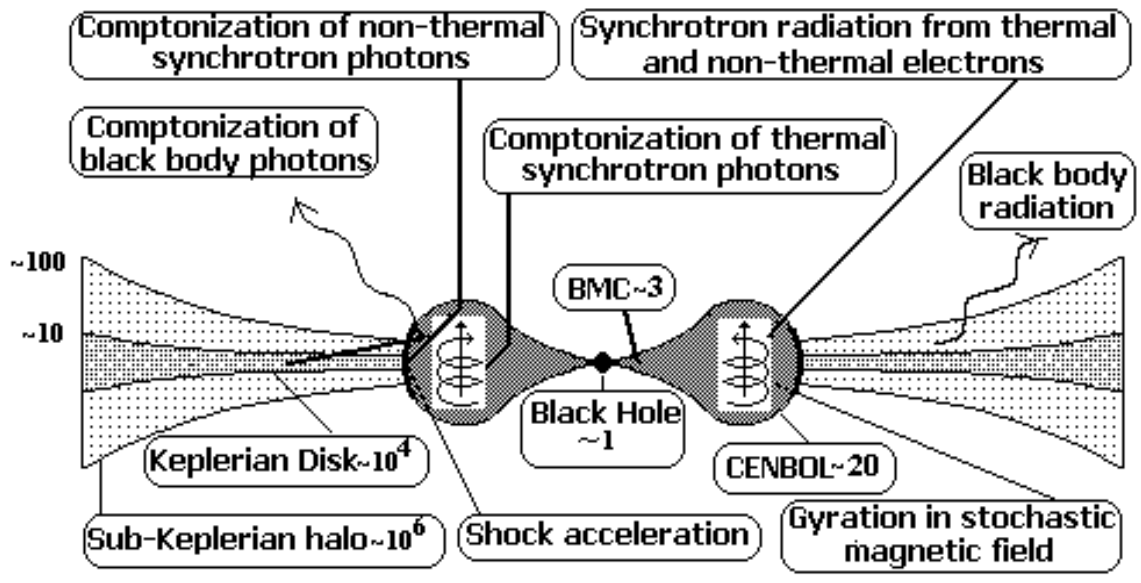

Figure 2: A cartoon diagram of the accretion flow with all the physical and spectral components. The dimension of different regions are in units of $r_{g}$, the radius of the black hole. The Keplerian flow is sandwiched by a sub-Keplerian halo both of which are terminated at an accretion shock. CENBOL represents the postshock region and is the source of thermal and non-thermal electrons. Soft photons from the Keplerian disk intercepted by the CENBOL and the synchrotron radiations from the thermal and the non-thermal electrons generated within CENBOL are inverse-Comptonized to higher energies (adapted from CM06).

gross properties of the observed QPOs can be tested with our solutions. For instance, Chakrabarti, Acharyya and Molteni (2004) showed, using simple cooling laws, that shocks oscillated both vertically and horizontally and the Fourier analysis of the emitted luminosity shows QPO peaks with similar characteristics as are in the observed radiation. From our model, the shocks are expected in hard states and thus we should have $v_{b}$ in hard states. Softer states are produced when accretion rate goes up and the shock propagates closer to the black hole resulting in higher QPO frequency, these are also observed. However, $r_{K e p}$ also propagates closer. Thus in softer states the frequency could be due to $v_{a}$ or $v_{b}$. Chakrabarti \& Manickam (2000) also showed that the post-shock photons only participate in the QPOs. As far as the 3:2 ratio of the QPO frequencies are concerned, we believe that this is due to spiral shocks which break from the axisymmetric shocks and have two or three arms. Details about these special types of QPOs are presented in another contribution (Chakrabarti and Wiita, this volume).

\section{Spectral Properties of the Shocked Two Component Flows}

So far, we discussed the properties of the oscillating shocks. However, in a large region of the parameter space spanned by specific energy and angular momentum of the fbw, the solution permits steady shocks in the accretion which must be included in spectral studies. Fortunately, shocks provide all the 'right' ingredients to generate a spectrum similar to the observed spectrum with a least number of free parameters. Shocks (say, at $r=r_{s}$ ) are produced due to the centrifugal force and the post-shock region $\left(r<r_{s}\right)$ is termed as the CENtrifugal pressure supported BOundary Layer (CENBOL) for the black hole as it behaves like a boundary layer where the fbw dissipates its thermal and kinetic energies. The CENBOL is puffed up and overlooks the pre-shock fbw 

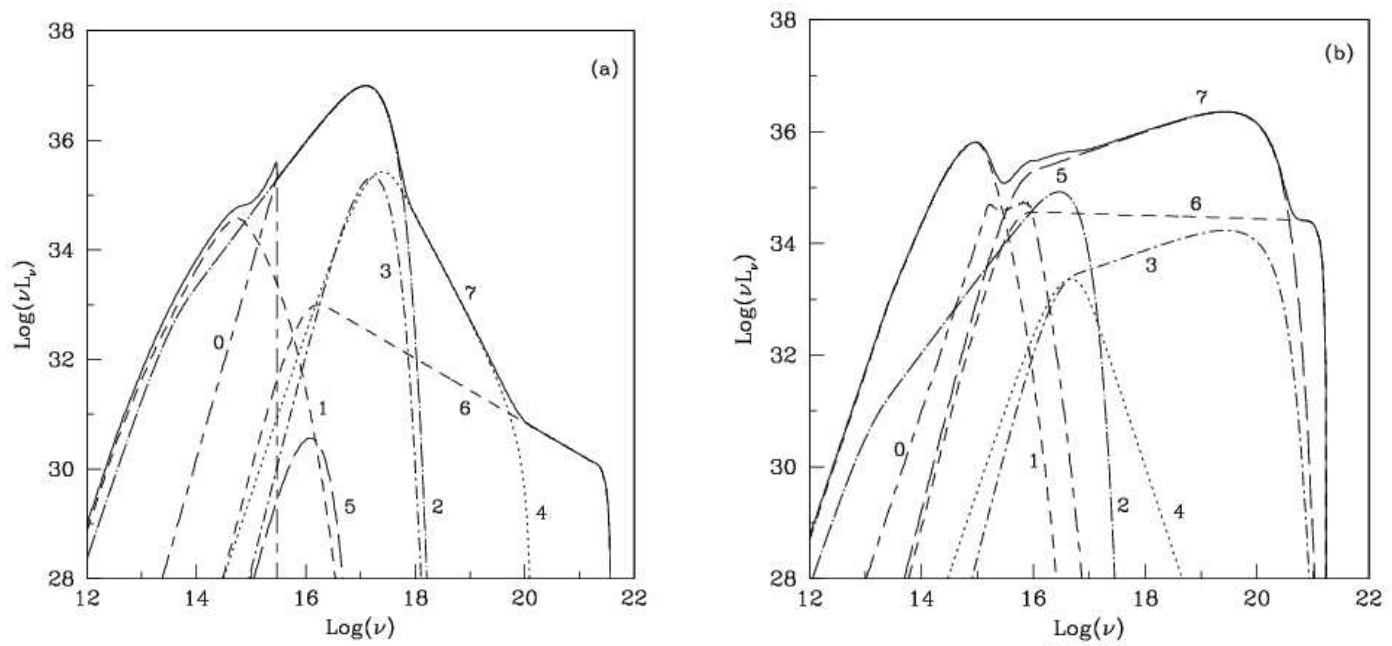

Figure 3: Nature of the spectrum from a two-component accretion flow around a $10 M_{\odot}$ black hole. In (a), the effect of bulk motion Comptonization is prominently seen (curve 4 contributing to the net spectrum curve 7) but the highest energy is achieved by the Comptonization of non-thermal electrons (curve 5 contributing to curve 7). We have drawn these curves using shock location $r_{s}=10$, percentage of power-law electrons $\zeta=0.1$, halo rate $\dot{m}_{h}=0.01$ and a disk rate $\dot{m}_{d}=1.0$. In (b), $\dot{m}_{h}=0.5, r_{s}=20.0, \zeta=0.04$ and $\dot{m}_{d}=0.01$ have been used. Here, the contribution due to BMC is insignifi cant. The description of other curves are given in the text.

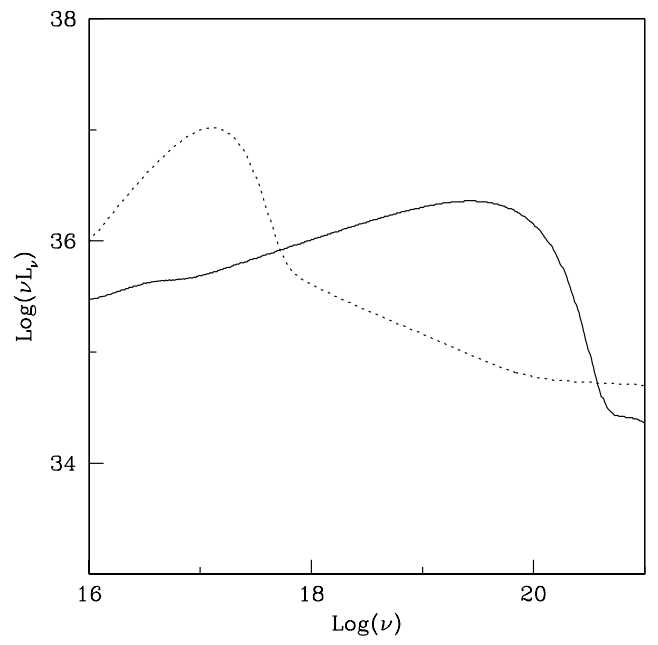

Figure 4: Typical spectra in the hard and the soft states intersecting at two energies, one at a few keV and the other at a few hundred $\mathrm{keV}$. 

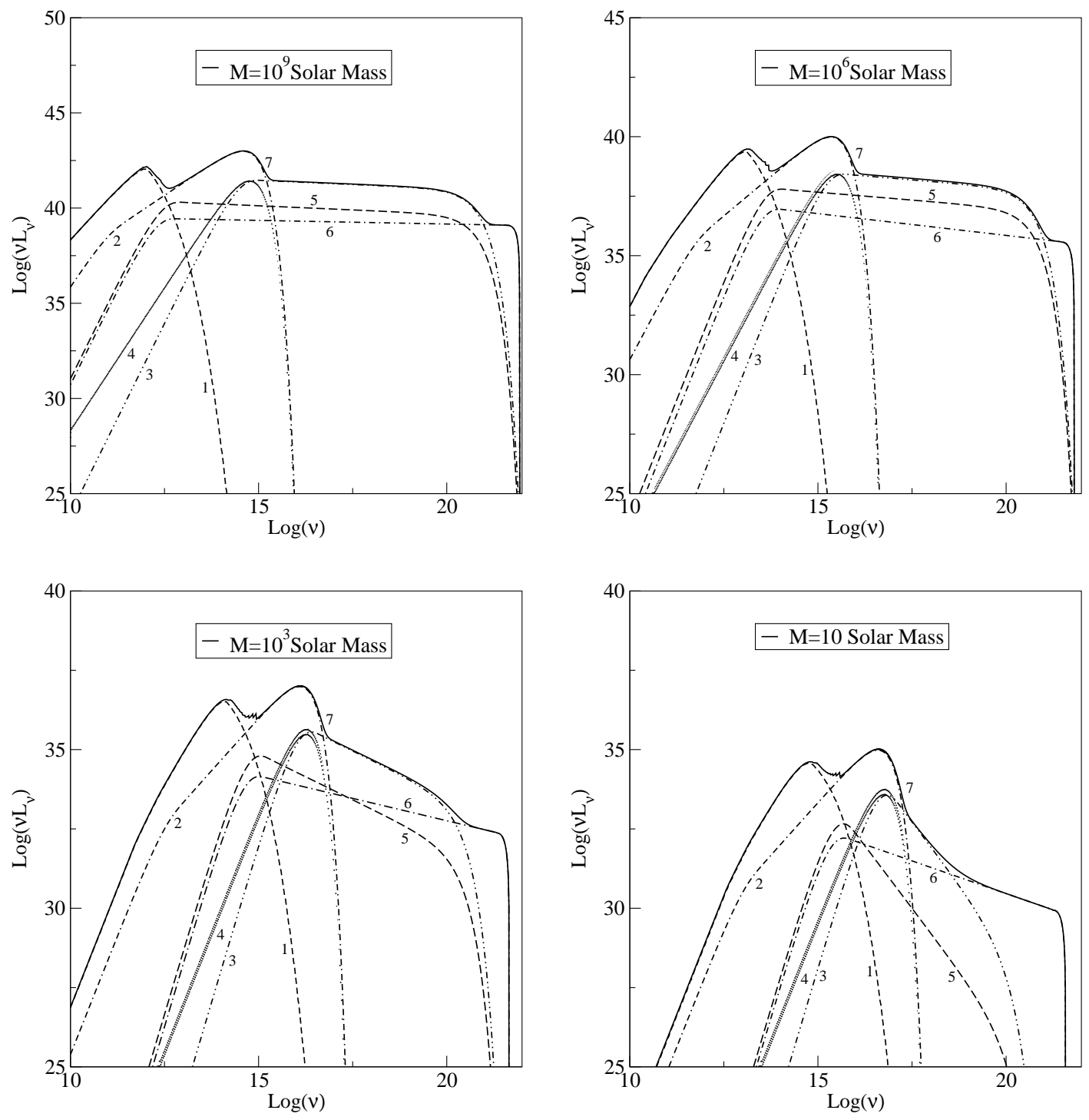

Figure 5: Spectra for a typical set of flow parameter when (a) $M=10^{9} M_{\odot}$, (b) $10^{6} M_{\odot}$, (c) $10^{3} M_{\odot}$ and (d) $10 M_{\odot}$ respectively.

$\left(r>r_{s}\right)$ to intercept the soft photons emitted from this region. The source of the soft photons could be a Keplerian disk or the synchrotron photons emitted in the sub-Keplerian fbw. In numerical simulations a smaller CENBOL (at $r_{i s s}$ ) is seen within the larger CENBOL due to turbulent effects (Samanta, Ryu and Chakrabarti, 2006). Fig. 2 shows a schematic diagram of the fbw configuration in our solution. The shock also accelerates the electrons and a fraction of them have power-law energy distribution. Mandal and Chakrabarti (2005) studied the effects of the shock accelerated electrons on the synchrotron soft-photons emitted by the sub-Keplerian electrons and Chakrabarti \& Mandal (2006, hereafter CM06) studied the spectrum in presence of both the Keplerian and the sub-Keplerian fbws. This is in effect extension of the earlier work by Chakrabarti \& Titarchuk (1995, here after CT95) after inclusion of the synchrotron photons and shock acceleration physics. They also fitted a few spectra of the black hole candidate Cyg X-1. 


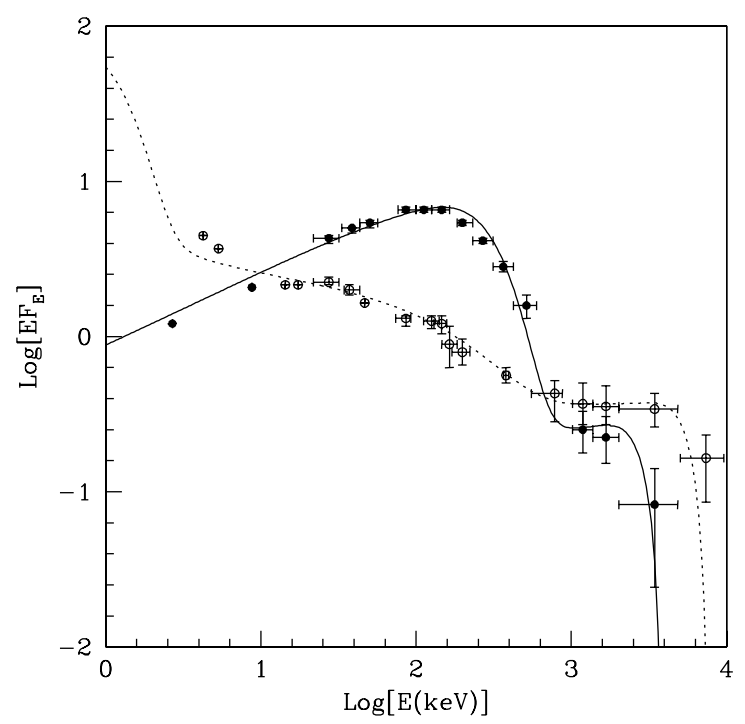

Figure 6: Fit of the observed spectra of Cyg X-1 in soft and hard states using the shocked accretion flow solution presented in this work. Open circles with error-bars represent the data points correspond to softstate and solid circles with error-bars represent the data points correspond to hard-state. The dotted and solid curves represent fi ts in the soft-state and the hard-states respectively. Note that there are two pivotal points one at a few $\mathrm{keV}$ and the other at a few hundred $\mathrm{keV}$. The hard-state is generated by a high sub-Keplerian rate and a low Keplerian rate, while the soft-state is generated by a low sub-Keplerian rate and a high Keplerian rate (CM06).

Figs. 3(a-b) show various contributions to the total spectrum. For illustration, we chose the black hole mass to be $M=10 M_{\odot}$. In Fig. 3a, the spectrum is 'soft' in the sense that the power is high at soft $\mathrm{X}$ ray region (CT95). The curves marked ' 0 ' indicates the synchrotron radiation from CENBOL, ' 1 ' indicates the synchrotron radiation from the pre-shock fbw, '2' black body radiation from the Keplerian disk, 3' represents the Comptonization of the intercepted black body photons by the CENBOL, ' 4 ' is due to Comptonization of the soft photons by the convergent fbw, ' 5 ' and ' 6 ' are the Comptonization of the synchrotron soft photons by the thermal electrons and non-thermal electrons respectively. Finally, the curve marked ' 7 ' represents the total spectrum. These curves are drawn for the accretion rate in the sub-Keplerian halo $m_{h}=0.01$, the accretion rate in the Keplerian disk $m_{l}=1$, the shock location $r_{s}=10$ and the fraction of power-law electrons $\zeta=0.1$. The accretion rates are measured in units of the Eddington rate and the shock location is measured in units of the Schwarzschild radius. Since the disk rate is very large compared to the halo rate, the post-shock fbw or the CENBOL region is cooled down and the usual thermal Comptonization becomes inefficient. But the Comptonization of the soft photons by the bulkmotion of the fbw shows a power-law tail extended up to a few hundreds of keV. Due to the shock acceleration process, a fraction of the electrons obey power-law distribution. The Comptonization spectrum of the synchrotron soft photons emitted by these non-thermal electrons shows a powerlaw nature extended up to a very high energy $(>100 \mathrm{MeV})$.

In Fig. 3b, we draw the spectral components for the spectrally 'hard' state in the sense that the emitted power is very high at hard $\mathrm{X}$ rays. Here, the parameters are $m_{h}=0.5, r_{s}=20.0$, $\zeta=0.04$ and $m_{l}=0.01$. The interpretations of various curves are the same as Fig. 3a. By varying 
the fbw parameters, we can reproduce two distinct states of a black hole in $\mathrm{X}$ rays as well as in $\gamma$-rays. In both cases, the high energy radiation is due to non-thermal electrons produced by the shock. Though in the $\mathrm{X}$ ray region these are called the soft and the hard states respectively, Ling and Wheaton (2005) call these states as the 'low $\gamma$ intensity state' and 'high $\gamma$ intensity state'. In Fig. 4 we present the net spectra for two cases: with the parameters $m_{h}=0.5, \quad m_{l l}=0.01, r_{s}=20$, $\zeta=0.04$ (solid) and $m_{h}=0.05, \quad m_{l}=1.0, r_{s}=10, \zeta=0.04$ (dotted). The first set corresponds to a high sub-Keplerian rate and low Keplerian rate which produces a hard state, and the second set has the rates other way around and produces a soft state (CT95). However, unlike CT95 cases, where there was only one so-called 'pivoting' point at around a few $\mathrm{keV}$, here we have an extra 'pivoting' point at a few hundred $\mathrm{keV}$ because of the inclusion of the synchrotron radiation. This is what is observed in black hole candidates (McConnell et al. 2000; 2002; Ling \& Wheaton 2005).

In Figs. 5(a-d), we show four spectra for black holes of masses $10^{9} M_{\odot}, 10^{6} M_{\odot}, 10^{3} M_{\odot}$ and $10 M_{\odot}$ respectively. The disk and halo accretion rates are, in units of the Eddington rate, 0.01 and 0.01 respectively for a strong shock (compression ratio $R=4$ ) located at $r_{s}=10$. Different curves have the same meanings as before. These should be the typical spectra of the $\sim$ quasars, $\sim$ milli-quasars, $\sim$ microquasars and $\sim$ nano-quasars respectively. The presence of two accretion rates (without increasing the number of parameters, since the viscosity parameter is absent) increases the types of spectra that one can have. This is particularly useful to explain the spectra of supermassive black holes.

In Figure 6, we superimpose the observed data (McConnell et al. 2000; McConnell et al. 2002) with our model fits. The important parameters of our models are halo rate $\left(m_{h}\right)$, disk rate $\left(i_{l}\right)$ and shock location $\left(x_{s}\right)$. In this Figure, the open circles and the solid circles with error-bars represent the soft-state data and the hard-state data of Cyg X-1. The soft-state and the hard-state data are fitted with a dotted curve and a solid curve respectively. The parameters for the soft state are $\dot{m}_{m}=0.3, \quad m_{l}=0.5, r_{s}=5$ and that for hard state are $m_{m}=1.47, \quad m_{l}=0.001, r_{s}=19.0$ respectively. In our solution, when Cyg X-1 changes its state from soft to hard, the Keplerian rate is decreased, the sub-Keplerian rate is increased and the shock recedes from the black hole. This is totally consistent with the two component advective disk paradigm presented in CT95 and Ebisawa, Titarchuk \& Chakrabarti (1996).

\section{References}

[1] Abramowicz, M. A. \& Chakrabarti, S. K., 1990, APJ, 350, 281

[2] Blandford, R. D. \& Payne, D., 1981, MNRAS, 194, 1033

[3] Chakrabarti, S. K. 1990, Theory of Transonic Astrophysical Flows (World Scientifi c: Singapore)

[4] Chakrabarti, S. K., 1994 in Proc. 17th Texas Symposium, Ed. H. Böhringer, E. Morfi 11 \& J. Trümper (New York Academy of Sciences, New York), p. 546.

[5] Chakrabarti, S. K., 1995, ApJ, 441, 576

[6] Chakrabarti, S. K., 1999, A\&A, 351, 185

[7] Chakrabarti, S. K., 2002, Frontiers in Astrophysics, Chakrabarti, S. K. (Ed.), 145, Allied Publishers (New Delhi)

[8] Chakrabarti, S. K., Acharyya, K. \& Molteni, D., 2004, A\&A 421, 1 
[9] Chakrabarti, S. K. \& Mandal, S., 2006, ApJ, 642, L49

[10] Chakrabarti, S. K. \& Manickam, S. G., 2000, ApJ 531, L41

[11] Chakrabarti, S. K. \& Nandi, A., 2000, Ind. J. Phys. 75B, 1

[12] Chakrabarti, S. K. \& Titarchuk, L. G., 1995, ApJ 455, 623 (CT95)

[13] Chakrabarti, S. K \& Wiita, P. J., 1992, ApJ, 387, L21

[14] Ebisawa, K., Titarchuk, L. G. \& Chakrabarti, S.K. 1996, PASJ, 48, 59

[15] Ford, H.C. et al., 1994, ApJ, 435, L27

[16] Ling, J. C. \& Wheaton, Wm. A., 2003, ApJ 584, 399

[17] Ling, J.C. \& Wheaton, Wm. A., 2005, ApJ 622, 492

[18] Ling, J. C. \& Wheaton, Wm. A., 2005, ChJAA Supplement 5, 80L

[19] Mandal, S. \& Chakrabarti, S. K., 2005, A\&A 434, 839

[20] McConnell et al., 2000, AP 543, 928

[21] McConnell et al., 2002, AP 572, 984

[22] Samanta, M.M., Ryu, D. Chakrabarti, S.K., 2006 (submitted)

[23] Shakura, N. I. \& Sunyaev, R. A. 1973, A\&A 24, 337

[24] Smith, D.M., Heindl, W.A., Markwardt, C.B. \& Swank, J.H., 2001, ApJ, 554, L41

[25] Smith, D.M., Heindl, W.A., \& Swank, J.H., 2002, ApJ, 569, 362 